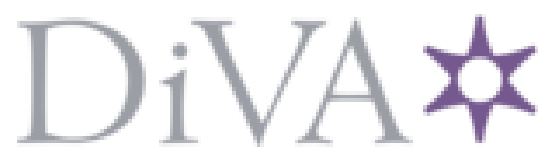

http://www.diva-portal.org

This is the published version of a paper presented at Oth International Conference on the European Energy Market (EEM), 20131.

Citation for the original published paper:

Tohidi, Y., Hesamzadeh, M. (2013)

Multi-national transmission planning using joint and disjoint solutions.

In:

N.B. When citing this work, cite the original published paper.

Permanent link to this version:

http://urn.kb.se/resolve?urn=urn:nbn:se:kth:diva-134308 


\section{Multi-national Transmission Planning Using Joint and Disjoint Solutions}

\author{
Yaser Tohidi \\ Electricity Market Research Group \\ KTH Royal Institute of Technology \\ Stockholm, Sweden \\ Email:tohi@kth.se
}

\author{
Mohammad R. Hesamzadeh \\ Electricity Market Research Group \\ KTH Royal Institute of Technology \\ Stockholm, Sweden \\ Email: mrhesa@kth.se
}

\begin{abstract}
Transmission systems have a critical role in wellfunctioning of liberalised electricity markets. Diversity in generation portfolio and improving system reliability are the main incentives for significant cross-border trades between Transmission System Operators (TSOs) in Europe. Nowadays, two ways are discussed for investment in additional transmission capacity by multiple TSOs: (1) Cooperative approach and (2) non-cooperative approache. In cooperative planning, the governing body looks for the highest overall social welfare while in non-cooperative approach, each TSO decides on his own transmission system based on his social welfare. The problem is that the outcome of these two approaches might be different. Each TSO tries to maximise his own social welfare and they are reluctant to act in a joint solution where they are losing some of their social welfare. In the context, they prefer to act strategically in order to maximise their social welfare. This paper evaluates the joint and disjoint solutions of the multi-national transmission planning. We propose a simultaneous-move game between the involved TSOs. The game is formulated as a one-shot mixedinteger linear programming problem. Then the joint solution is developed by merging all TSOs in one TSO responsible for transmission planning in all TSOs' regions. The joint solution is formulated as a mixed-integer linear programming and used as the benchmark in our studies.
\end{abstract}

Index Terms-Transmission planning, Multi-national TSOs, Joint and Disjoint solutions

\begin{tabular}{|c|c|}
\hline & \\
\hline$A_{n k}$ & Element of network incidence matrix, 1 when bus \\
\hline & $\begin{array}{l}n \text { is the sending bus of line } k,-1 \text { when bus } n \text { is } \\
\text { the receiving bus of line } j \text {, and } 0 \text { otherwise }\end{array}$ \\
\hline$u_{k}$ & Binary decision variable of candidate line $\mathrm{k}$ \\
\hline$c_{n g}$ & Fuel cost of old unit $g$ at bus $n$ \\
\hline FC & Fuel cost \\
\hline$G_{n}$ & Number of generating units at bus $n$ \\
\hline IC & Investment cost \\
\hline $\operatorname{Inv} v_{k}$ & Investment cost of candidate line $\mathrm{k}$ \\
\hline $\mathrm{L}$ & Number of load levels \\
\hline$L C_{n l s}$ & Load curtailment in load level 1 at bus $n$ \\
\hline $\operatorname{Load}_{n l}$ & Load value in load level 1 at bus $n$ \\
\hline M & Penalty factor of disjunctive approach \\
\hline$n_{k}$ & Life time of candidate line $\mathrm{k}$ \\
\hline NB & Number of buses \\
\hline CL & Number of candidate lines \\
\hline
\end{tabular}

\begin{tabular}{|c|c|}
\hline EL & Number of existing lines \\
\hline$p_{k l}^{e x}$ & $\begin{array}{l}\text { Continues variable of power flow of existing line } \\
\mathrm{k} \text { in load level } 1\end{array}$ \\
\hline$p_{k l}^{c a n}$ & $\begin{array}{l}\text { Continues variable of power flow of candidate } \\
\text { line } \mathrm{k} \text { in load level } 1\end{array}$ \\
\hline & Capacity of existing line $\mathrm{k}$ \\
\hline $\bar{p}_{k}^{c a n}$ & Capacity of candidate line $\mathrm{k}$ \\
\hline$p_{n g l}$ & $\begin{array}{l}\text { Continues variable of power generation of gener- } \\
\text { ation unit } g \text { at bus } n \text { in load level } 1\end{array}$ \\
\hline $\bar{p}_{n g}$ & Capacity of generating unit $\mathrm{g}$ at bus $\mathrm{n}$ \\
\hline $\mathrm{r}$ & Interest rate \\
\hline $\mathrm{LCC}$ & Load curtailment cost \\
\hline$t_{l}$ & Duration of load level 1 \\
\hline$V O L L_{n}$ & Value of lost load at bus $n$ \\
\hline$x_{k}$ & Reactance of line $\mathrm{k}$ \\
\hline $\mathrm{Z}$ & $\begin{array}{l}\text { Number of different zones operating by different } \\
\text { TSOs }\end{array}$ \\
\hline & Voltage phase angle of bus $n$ in load level 1 \\
\hline
\end{tabular}

\section{INTRODUCTION}

In liberalised electricity markets, transmission system operators (TSOs) were formed to provide access for all market participants for trading electrical energy and operate the system within an acceptable reliability level. However, transmission grids have limited transmission capacity. TSOs continuously plan for their future transmission capacity in order to keep the electricity network and electricity markets well-functioning. Transmission planning is one of the responsibilities of TSOs and it is the problem of finding best technology to invest in, best time for investment, and best location for investment. In vertically-integrated electricity industry, the transmission planning problem can be formulated as an optimisation problem. The transmission planner minimises the investment costs subject to a set of technical constraints. In the liberalised electricity markets, the optimisation problem is modified by adding the social welfare to the objective function of the transmission planning problem, [1-5]. Each transmission system operator maximises the social welfare of his electricity network. Crossborder trading is a current and ongoing issue in different active markets in Europe. TSOs have found out that there is an opportunity in cross-border trades originated by diversity of 
generating capacities and complementary generation sources located in different places. Diversity of generating capacities means it might happen that a region with low generating capacity is adjacent to a region with high generating capacity. Also, difference between peak load days in these areas make a good opportunity for generating units to participate in markets of each other. Realisation of this benefits is limited by the capacity of transmission grids. An economically efficient trading between different multi-national markets needs more multi-national transmission planning.

There are few papers in the literature which deal with the multi-national transmission planning.

The problem of multi-national transmission planning can be tackled in two ways: (1) cooperative or joint approach, and (2) non-cooperative or disjoint approach.

In cooperative planning, the governing body looks for the highest overall social welfare while in non-cooperative approach, each TSO decides on his own transmission system based on his social welfare which results to a set of transmission investments solutions for each TSO. The problems with the cooperative approach is twofold: (1) It is less likely to happen, and (2) Sharing of the transmission investment cost is difficult. But this approach leads to the highest social welfare.

There are some publications about the allocation cost of cooperative transmission expansion planning [6,7]. They determine loosers and winners of the cooperative transmission plan and try to share the transmission investment cost accordingly. However, even if winners of cooperative plan are willing to contribute in the investment cost in the area hosting the infrastructure, the hosting area still can block the investment by for example interference in the permitting process. Thus, the compensation mechanism should be designed as a regulatory framework for the usage of the transmission corridor.

The non-cooperative approach is most likely to happen but it results in less social welfare. The non-cooperating transmission planning and its comparison with cooperative transmission planning have not been addressed extensively in literature. In [8], a game between transmission investors is considered, with the assumption of privately owned TSOs. It does not evaluate the difference between the cooperative and non-cooperative approaches. Reference [9] compares these two approaches and concludes that they result to different optimum investment capacities but its variables are continuous which is not the case in transmission expansion planning formulation.

The remainder of the paper is organized as follows. Firstly, mathematical models for both the cooperative and non-cooperative approach are presented in sections II. Then, the models are tested on two examples and the results are compared between the two approaches in section III. Section IV concludes the paper.

\section{Mathematical Formulation}

The mathematical formulation of transmission expansion planning problem is developed in this section. The objective function of the problem is to maximize the social welfare which is the difference between benefits and investment costs.
Social welfare maximization can be considered as the social cost minimization, the objective function is composed of three terms: (1) investment cost (IC) (2) fuel cost (FC) and (3) load curtailment cost (LCC). For effective modeling of the investment cost in the planning horizon, it is replaced with the correlated annual equivalent value as stated in (2). The decision variable of a new line $(u)$ is 1 if it is planned to be invested.

$$
\begin{gathered}
\min C O S T=I C+F C+L C C \\
I C=\sum_{k=1}^{C L} u_{k} \times I n v_{k} \times \frac{r(1+r)^{n_{k}}}{(1+r)^{n_{k}}-1} \\
F C=\sum_{n=1}^{N B} \sum_{l=1}^{L} t_{l} \times \sum_{g=1}^{G_{n}} c_{n g} \times p_{n l g} \\
L C C=\sum_{n=1}^{N B} \sum_{l=1}^{L} t_{l} \times V O L L_{n} \times L C_{n l}
\end{gathered}
$$

Equality and inequality constraints of the problem consist of capacity of units (constraint (5)), capacity of existing lines (constraint (6)), capacity of candidate lines (constraint (7)), second Kirchhof's law for existing lines (constraint (8)), second Kirchhof's law for candidate lines (constraint (9)), buslevel load balance (constraint (10)), and the reference bus constraint ((constraint (11)).

$$
\begin{gathered}
p_{n g l} \leq \bar{p}_{n g l} \quad n=1 \cdot N B, g=1 \cdot G_{n}, l=1 \cdots L \\
-\bar{p}_{k}^{e x} \leq p_{k l}^{e x} \leq \bar{p}_{k}^{e x} \quad k=1 \cdot E L, l=1 \cdot L \\
-\bar{p}_{k}^{c a n} \times u_{k} \leq p_{k l}^{c a n} \leq u_{k} \times \bar{p}_{k}^{c a n} \quad k=1 \cdot C L, l=1 \cdot \cdots L \\
p_{k l}^{e x}=\frac{1}{x_{k}} \sum_{n=1}^{N B} A_{n k} \times \delta_{n l} \quad k=1 \cdot E L, l=1 \cdots L \\
p_{k l}^{c a n}=u_{k} \times \frac{1}{x_{k}} \times\left(\delta_{i l}-\delta_{j l}\right) \quad k=1 \cdot C L, k \leftarrow(i, j), l=1 \cdot \cdot L \\
\text { Load }_{n l}=L C_{n l}+\sum_{g=1}^{G_{n}} p_{n g l}-\sum_{k=1}^{O L} A_{n k} \times p_{k l}^{e x} \\
\sum_{k=1, i_{k}=n}^{C L} p_{k l}^{c a n}+\sum_{k=1, j_{k}=n}^{C L} p_{k l}^{c a n} \\
n=1 \cdot N B, l=1 \cdots L
\end{gathered}
$$


Product of variables in second Kirchhof's law for candidate lines (constraint (9)) makes the problem nonlinear. This makes the optimisation problem hard to solve. This nonlinearity can be avoided by using an equivalent disjunctive form proposed in [10]. As expressed in (12), the capacity constraint for candidate lines is now replaced by two linear inequalities using a large enough $M$.

$$
\begin{array}{r}
-M\left(1-u_{k}\right) \leq p_{k l}^{c a n}-\frac{1}{x_{k}} \times\left(\delta_{i l}-\delta_{j l}\right) \leq M\left(1-u_{k}\right) \\
k=1 \cdot C L, k \leftarrow(i, j), l=1 \cdots L
\end{array}
$$

When a candidate circuit binary variable is set to zero, no flow will go through the line and if it is set to one, the flow will obey the second Kirchhoff's law equation.

The model developed above is a mixed-integer linear programming problem.

We use this formulation for cooperative approach where all TSOs cooperative and merge to one entity looking for the highest overall social welfare.

In the non-cooperative approach, each TSO decides about his own candidate lines and maximizes his own social welfare. We assume that the dispatch formulation is the same for all involved TSOs. This concept is illustrated in Figure 1.

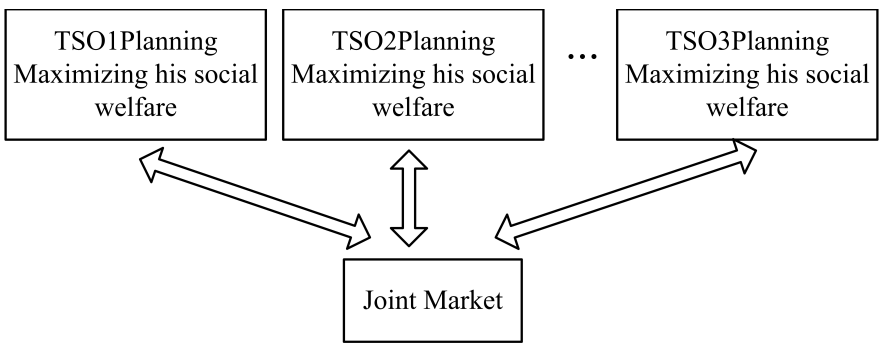

Fig. 1: Non-cooperative transmission planning structure.

Each TSO maximises his own social welfare (which can be considered as the social cost minimization) taking into account the strategic reactions of other TSOs. The objective function of each TSO $\mathrm{z}$ is set put in (13)-(16). The constraints are set out in (17)-(19).

$$
\min \operatorname{COST}_{z}=I C_{z}+F C_{z}+L C C_{z} \quad z=1 \cdot Z
$$

$$
\begin{gathered}
I C_{z}=\sum_{k=1}^{C L_{z}} u_{k} \times I n v_{k} \times \frac{r(1+r)^{n_{k}}}{(1+r)^{n_{k}}-1} \quad z=1 \cdots Z \\
F C_{z}=\sum_{n=1}^{N B_{z}} \sum_{l=1}^{L} t_{l} \times \sum_{g=1}^{G_{n z}} c_{n g} \times p_{n l g} \quad z=1 \cdots Z \\
L C C_{z}=\sum_{n=1}^{N B_{z}} \sum_{l=1}^{L} t_{l} \times V O L L_{n} \times L C_{n l} \quad z=1 \cdots Z
\end{gathered}
$$

$$
\begin{gathered}
\min C O S T=F C+R C \\
F C=\sum_{n=1}^{N B} \sum_{l=1}^{L} t_{l} \times \sum_{g=1}^{G_{n}} c_{n g} \times p_{n l g} \\
R C=\sum_{n=1}^{N B} \sum_{l=1}^{L} t_{l} \times V O L L_{n} \times L C_{n l}
\end{gathered}
$$

The strategic reaction between different TSO's can be formulated as a Nash equilibrium problem. In game theory, the Nash equilibrium is the set of strategy choices in a noncooperative game involving two or more players, in which each player knows the strategies of the other players, and when players choose their strategies, no player can benefit by changing his or her strategy while the other players keep theirs unchanged [11].

Suppose $\left(x_{1}, \ldots, x_{m}\right)$ is the decision vector of $m$ players with the feasible set of $X$ and $f_{i}\left(x_{i}\right)$ is the objective function of the $i$ th player. It is assumed that the players (the TSOs) seek to minimize their objective functions (i.e., to minimize their cost).

All the players are from the same level and they must reveal their strategies simultaneously. So, the solution is the Nash equilibrium which is defined as the array $\left(x_{1}^{*}, \ldots, x_{m}^{*}\right)$ which satisfies

$$
\begin{aligned}
& f_{i}\left(x_{1}^{*}, \ldots, x_{i-1}^{*}, x_{i}^{*}, x_{i+1}^{*}, \ldots, x_{m}^{*}\right) \leq \\
& \qquad f_{i}\left(x_{1}^{*}, \ldots, x_{i-1}^{*}, x_{i}, x_{i+1}^{*}, \ldots, x_{m}^{*}\right) \\
& \text { for } \quad \text { any } \quad\left(x_{1}^{*}, \ldots, x_{i-1}^{*}, x_{i}, x_{i+1}^{*}, \ldots, x_{m}^{*}\right) \epsilon X \quad \text { and }
\end{aligned}
$$
$i=1,2, \ldots, m$.

We will not go through the mathematical modeling of this problem and we solve the non-cooperative transmission planning with force enumeration approach in this paper. It means that we assess all options for transmission planning and get the payoffs for each TSO in each combination of candidate units.

\section{Simulation Results}

To compare the two cooperative and non-cooperative approaches for transmission expansion planning in the context of multiple TSOs, a network consisting of 3 TSOs is considered as depicted in Figure 3. Nodes 1 to 3 are located in TSO1 area, Nodes 4 to 6 are located in TSO2 area, and Nodes 7 to 9 are located in TSO3 area. Also, the link between node 2 and node $4\left(u_{1}\right)$ is located in TSO1, link between node 2 and node $9\left(u_{3}\right)$ in TSO3s area, and the link between node 3 and node $6\left(u_{2}\right)$ in TSO2s area. The transmission line data is given in Table I.

Each TSO is modelled based on the designed system in [12]. The existing lines are drawn with solid lines while the three candidate lines are presented with the dashed lines. The data for this system are set out in Table I to Table IV. Interest 
TABLE I: Generation Data

\begin{tabular}{c|c|c}
\hline Generating Unit & Operating Cost (\$/MWh) & Max. Capacity (MW) \\
\hline \hline$G 1$ & 42 & 100 \\
\hline$G 2$ & 35 & 200 \\
\hline$G 3$ & 42 & 100 \\
\hline$G 4$ & 35 & 200 \\
\hline$G 5$ & 42 & 100 \\
\hline$G 6$ & 35 & 200 \\
\hline
\end{tabular}

TABLE II: Demand Data

\begin{tabular}{c|c|c}
\hline Bus\# & Demand (MW) & Value of Lost Load (\$/MWh) \\
\hline \hline 1 & 75 & 10,000 \\
\hline 3 & 100 & 10,000 \\
\hline 4 & 225 & 10,000 \\
\hline 6 & 300 & 10,000 \\
\hline 7 & 37.5 & 10,000 \\
\hline 9 & 50 & 10,000 \\
\hline
\end{tabular}

TABLE III: Transmission Network Data

\begin{tabular}{c|c|c|c|c}
\hline Line\# & Owner & To-From & Reactance (Ohm) & Limit (MW) \\
\hline \hline 1 & TSO1 & $1-2$ & 0.002 & 134 \\
\hline 2 & TSO1 & $2-3$ & 0.002 & 147 \\
\hline 3 & TSO1 & $1-3$ & 0.002 & 25 \\
\hline 4 & TSO2 & $4-5$ & 0.002 & 134 \\
\hline 5 & TSO2 & $5-6$ & 0.002 & 147 \\
\hline 6 & TSO2 & $4-6$ & 0.002 & 25 \\
\hline 7 & TSO3 & $7-8$ & 0.002 & 134 \\
\hline 8 & TSO3 & $8-9$ & 0.002 & 147 \\
\hline 9 & TSO3 & $7-9$ & 0.002 & 25 \\
\hline 10 & TSO1 & $2-4$ & 0.002 & 50 \\
\hline 11 & TSO3 & $3-9$ & 0.002 & 60 \\
\hline
\end{tabular}

TABLE IV: Candidate Lines Data

\begin{tabular}{c|c|c|c|c|c|c}
\hline Binary Variable & Owner & To-From & Reactance $(\mathrm{Ohm})$ & Limit (MW) & Investment Cost (\$) & Lifetime (Years) \\
\hline \hline$u_{1}$ & TSO1 & $2-4$ & 0.002 & 50 & 2,000 & 20 \\
\hline$u_{2}$ & TSO2 & $3-6$ & 0.002 & 50 & 2,000 & 20 \\
\hline$u_{3}$ & TSO3 & $2-9$ & 0.002 & 60 & 1,000 & 20 \\
\hline
\end{tabular}

rate is assumed to be 5\%. The CPLEX solver in GAMS is used to solve the optimization problem.

Table V and VI present the social cost under each combination of new lines and for each TSO (three candidate lines result in eight different combinations for future investments). In Table $\mathrm{V}, u_{3}$ equals to 0 and it is equal to 1 in Table VI. Note that in non-cooperative approach, each TSO can decide about the candidate line in his area of responsibility. For example, if decision variable of TSO1 equals to (0) 1 , it means that TSO1 decides (not) to invest in the line between nodes 2 and 4 . The results of these two approaches are presented in Table IV.

The optimum solution of cooperative approach is a new line between TSO1 and TSO2 with a total cost of $1.4394 \times 10 \$$.

On the other hand, the Nash equilibrium of the noncooperative approach is "not to invest". The Nash solution of "not to invest" is the equilibrium point between all three TSOs. At this equilibrium, no TSO can benefit by moving unilaterally while the other players stay at their equilibrium solution. As you can see in Table VII, total social cost of the whole area in Nash equilibrium point is higher than the social cost of the whole area in cooperative approach. The difference between these two approaches can be explained as follows: In cooperative approach, the investment cost of the new line between TSO1 and TSO2 is being compensated with the reduction of load curtailment in $\mathrm{TSO} 2$ and the benefit of this investment is mainly for TSO2 while the owner and investor of this new line is TSO1. The result of non-cooperative approach shows that TSO1 has no incentive to invest in this line. It should be noted that welfare loss for TSO1 in the cooperative approach compare to non-cooperative approach is smaller than the welfare gain of TSO2.

A compensating mechanism in favor of TSO1 can make the result of non-cooperative approach the same as cooperative approach result. Note that extra generation capacity of TSO3 can find a flow to TSO2 load buses through the line between TSO1 and TSO2. This means that the free riding effect should also be considered in designing the compensation mechanism.

\section{CONCLUSION}

Transmission expansion planning in the context of multiple TSOs can be approached using cooperative or non-cooperative 
TABLE V: Social Cost $(\$)$ for Each TSO $\left(u_{3}=0\right)$

\begin{tabular}{|c|c|c|}
\hline$u_{3}=0$ & $u_{1}=0$ & $u_{1}=1$ \\
\hline \multirow{4}{*}{$u_{2}=0$} & $S C_{1}=5.3508 \times 10^{7}$ & $S C_{1}=5.358 \times 10^{7}$ \\
& $S C_{2}=1.54042 \times 10^{10}$ & $S C_{2}=1.43168 \times 10^{10}$ \\
& $S C_{3}=2.37 \times 10^{7}$ & $S C_{3}=2.37 \times 10^{7}$ \\
\cline { 2 - 3 } & $T O T A L S C=1.54814 \times 10^{10}$ & $T O T A L S C=1.4389 \times 10^{10}$ \\
\hline \multirow{4}{*}{$u_{2}=1$} & $S C_{1}=5.6566 \times 10^{7}$ & $S C_{1}=5.5113 \times 10^{7}$ \\
& $S C_{2}=1.54055 \times 10^{10}$ & $S C_{2}=1.43181 \times 10^{10}$ \\
\cline { 2 - 3 } & $S C_{3}=2.37 \times 10^{7}$ & $S C_{3}=2.37 \times 10^{7}$ \\
\cline { 2 - 3 } & $T O T A L C C=1.54858 \times 10^{10}$ & TOTALSC $=1.4397 \times 10^{10}$ \\
\hline
\end{tabular}

TABLE VI: Social Cost $(\$)$ for Each TSO $\left(u_{3}=1\right)$

\begin{tabular}{|c|c|c|}
\hline$u_{3}=1$ & $u_{1}=0$ & $u_{1}=1$ \\
\hline \multirow{4}{*}{$u_{2}=0$} & $S C_{1}=5.42724 \times 10^{7}$ & $S C_{1}=5.358 \times 10^{7}$ \\
& $S C_{2}=1.54042 \times 10^{10}$ & $S C_{2}=1.43168 \times 10^{10}$ \\
& $S C_{3}=2.4227 \times 10^{7}$ & $S C_{3}=2.4227 \times 10^{7}$ \\
\cline { 2 - 3 } & $T O T A L S C=1.54827 \times 10^{10}$ & $T O T A L S C=1.43946 \times 10^{10}$ \\
\hline \multirow{4}{*}{$u_{2}=1$} & $S C_{1}=5.733 \times 10^{7}$ & $S C_{1}=5.5877 \times 10^{7}$ \\
& $S C_{2}=1.54055 \times 10^{10}$ & $S C_{2}=1.43181 \times 10^{10}$ \\
& $S C_{3}=2.4227 \times 10^{7}$ & $S C_{3}=2.4227 \times 10^{7}$ \\
\cline { 2 - 3 } & $T O T A L S C=1.5487 \times 10^{10}$ & $T O T A L S C=1.43982 \times 10^{10}$ \\
\hline
\end{tabular}

TABLE VII: Comparison of the Results of Cooperative and Non-cooperative Approaches

\begin{tabular}{|c|c|c|}
\hline Approaches & Final solution & Total $\operatorname{Cost}(\$)$ \\
\hline Cooperative & $u=(1,0,0)$ & $1.4394 \times 10^{10}$ \\
\hline Non-cooperative & $u=(0,0,0)$ & $1.54814 \times 10^{10}$ \\
\hline
\end{tabular}

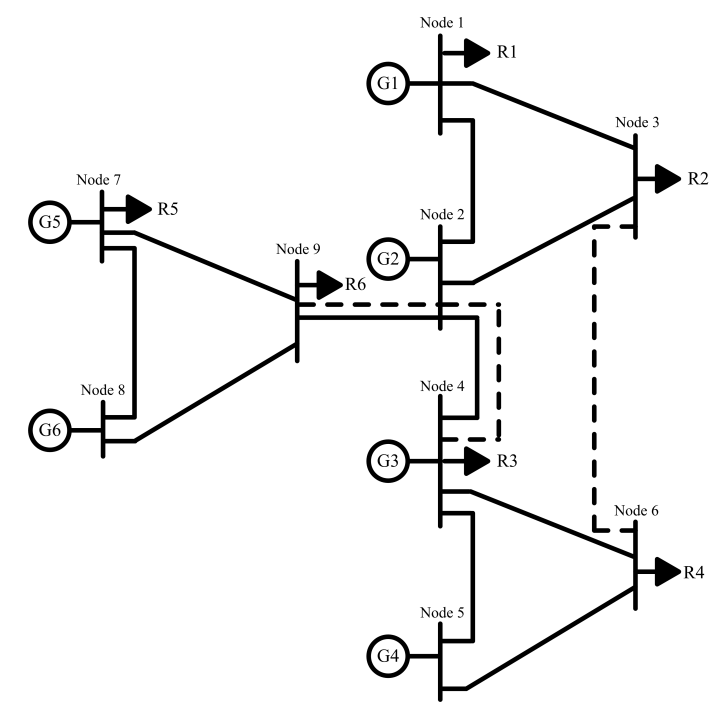

Fig. 2: Single-line diagram of the network consisting of three TSOs.

approaches. In cooperative approach, several TSOs cooperate in transmission planning with the aim to maximize overall social welfare but non-cooperative approach represents a game played by different TSOs deciding on investments in their area of responsibility at the first level and take part in a same market at the second level. This paper emphasized on the difference between cooperative and non-cooperative transmission planning approaches and also the importance of designing correct compensation mechanisms for TSOs to move toward cooperative solution. This is illustrated in this paper using three interconnected TSOs. The mathematical formulation developed in this paper is based on the MIP format and the model was effectively solved using a commercial MIP solver. The simulation results reveal that the outcome of two approaches can be different and non-cooperative TSOs may not reach to the best overall social welfare scheme. However, an appropriate compensation mechanism could be implemented to make equilibrium point of the game the same as the result of cooperative approache. In this way, TSOs shall receive compensation for hosting cross-border flows of electricity on their networks. Moreover, given that most of the transmission costs are recovered from load, compensations cause incentives for new line investment on the hosting TSO that generates and exports power to another TSO. For this to be realised, it's important that regulatory bodies provide necessary frameworks for the TSOs to operate in.

\section{REFERENCES}

[1] A. K. David and F. S. Wen, "Transmission planning and investment under competitive electricity market environment," in Proc. IEEE Power Eng. Soc. Summer Meeting, Jul. 2001, vol. 3, pp. 1725-1730.

[2] H. Chao, F. X. Li, L. H. Trinh, J. P. Pan, M. Gopinathan and D. J. Pillo, "Market based transmission planning considering reliability and economic performances," Int. Conf. on Probabilistic Methods Applied to Power Syst., pp. 557-562, Sep. 2004.

[3] N. Yang and F. S. Wen, "A chance constrained programming approach to transmission system expansion planning," Electric Power Systems Research, vol. 75, pp. 171-177, Aug. 2005.

[4] A. S. D. Braga and J. T. Saraiva, "A multiyear dynamic approach for transmission expansion planning and long-term marginal costs computation," IEEE Trans. Power Syst., vol. 20, pp. 1631-1639, Aug. 2005. 
[5] G. B. Shrestha and P. A. J. Fonseka, "Congestion-driven transmission expansion in competitive power markets," IEEE Trans. Power Syst., vol. 19, pp. 1658-1665, Aug. 2004.

[6] D. Gately, "Sharing the gains from regional cooperation: a game theoretic application to planning investment in electric power," International Economic Review, vol. 15, no.1, pp. 195-208, Feb. 1974.

[7] P. Joskow and J. Tirole, "Merchant transmission investment," Journal of Industrial Economics, vol. 53, no. 2, pp. 233264, June 2005.

[8] J. Contreras and F. Wu, "Coalition formation in transmission expansion planning," Power Systems, IEEE Transactions on, vol. 14, no. 3, pp. 1144 1152, August 1999.

[9] P. Buijs and R. Belmans, "Transmission investments in a multilateral context," Power Systems, IEEE Transactions on, vol. 27, no. 1, pp. 475 - 483, Feb. 2012.

[10] L. Bahiense, G. C. Oliveira, M. Pereira, and S. Granville, "A mixed integer disjunctive model for transmission network expansion," IEEE Trans. Power Syst., vol. 16, no. 3, pp. 560-565, Aug. 2001.

[11] J. Nash, "Non-Cooperative Games," The Annals of Mathematics, vol. 54, no. 2, pp. 286-295, 1951.

[12] M. R. Hesamzadeh, D. R. Biggar, N. Hosseinzadeh, and P. J. Wolfs, "Transmission Augmentation with mathematical modeling of market power and strategic generation expansionPart I,' IEEE Trans. Power Syst., vol. 26, no. 4, pp. 2040 - 2048, Nov. 2011.

Yaser Tohidi received the B.Sc. degree in electrical engineering in 2008 and the M.Sc. degree in Power Systems in 2011. He continued his studies at KTH Royal Institute of Technology, Sweden, and now he is working toward his Ph.D. degree. He is currently engaged in transmission planning in market environment.

Mohammad Hesamzadeh (M'10) received the $\mathrm{PhD}$ degree from Swinburne University of Technology, Australia, and currently pursuing the Docent degree at KTH Royal Institute of Technology, Sweden. He did his postdoctoral fellowship at KTH. He is currently an assistant professor in the Department of Electric Power Systems, School of Electrical Engineering at KTH. His special fields of interests include Power Systems Planning and Design, Economics of Wholesale Electricity Markets, and Mathematical Modelling and Computing. Dr. Hesamzadeh is a member of International Association for Energy Economics (IAEE) and a member of the CIGRE National Committee in Sweden. 